\title{
Determinants of Virulence and In Vitro Development Colocalize on a Genetic Map of Setosphaeria turcica
}

\author{
Santiago X. Mideros, ${ }^{\dagger}$ Chia-Lin Chung, Tyr Wiesner-Hanks, Jesse A. Poland, Dongliang Wu, Ariel A. Fialko, \\ B. Gillian Turgeon, and Rebecca J. Nelson
}

First author: University of Illinois at Urbana-Champaign, Urbana 61801; second author: National Taiwan University, Taipei, 10617, Taiwan; and third, fourth, fifth, sixth, seventh, and eighth authors: School of Integrative Plant Science, Cornell University, Ithaca, NY 14853.

Current address of J. A. Poland: Kansas State University, Manhattan 66506.

Accepted for publication 24 September 2017.

\begin{abstract}
Generating effective and stable strategies for resistance breeding requires an understanding of the genetics of host-pathogen interactions and the implications for pathogen dynamics and evolution. Setosphaeria turcica causes northern leaf blight (NLB), an important disease of maize for which major resistance genes have been deployed. Little is known about the evolutionary dynamics of avirulence $(A V R)$ genes in S. turcica. To test the hypothesis that there is a genetic association between avirulence and in vitro development traits, we (i) created a genetic map of $S$. turcica, (ii) located candidate AVRHt1 and AVRHt2 regions, and (iii) identified genetic regions associated with several in vitro development traits. A cross was generated

between a race 1 and a race $23 \mathrm{~N}$ strain, and 221 progeny were isolated. Genotyping by sequencing was used to score 2,078 single-nucleotide polymorphism markers. A genetic map spanning 1,981 centimorgans was constructed, consisting of 21 linkage groups. Genetic mapping extended prior evidence for the location and identity of the AVRHt1 gene and identified a region of interest for $A V R H t 2$. The genetic location of $A V R H t 2$ colocalized with loci influencing radial growth and mycelial abundance. Our data suggest a trade-off between virulence on $H t 1$ and $H t 2$ and the pathogen's vegetative growth rate. In addition, in-depth analysis of the genotypic data suggests the presence of significant duplication in the genome of $S$. turcica.
\end{abstract}

Plant resistance is often used as a crop protection strategy but major resistance $(R)$ genes are frequently overcome as pathogen populations evolve to avoid the recognition processes that enable plants to mount effective resistance responses (McDonald and Linde 2002). The durability of resistance depends, among other things, on the fitness cost of overcoming resistance; the vigor and reproductive potential of pathogen variants that are able to overcome specific $R$ genes will influence the long-term performance of varieties carrying those $R$ genes (Leach et al. 2001).

There is evidence from a variety of pathosystems that the ability to overcome resistance is associated with reductions in fitness (Huang et al. 2010). Such "costs of virulence" vary, presumably because there are diverse physiological challenges associated with modifying pathogen effectors to avoid recognition by the host $R$ gene or its guard molecules. Modeling approaches have suggested that this cost has implications for the stability of virulence and aggressiveness traits in populations with and without selection pressure for virulence (Pietravalle et al. 2006).

We sought to explore the genetic relationships among pathogen effectors that provide incompatibility on major disease resistance and fitness-related traits for an important maize pathogen in order to gain insights into the trade-offs involved in overcoming $R$ genes. Genetic mapping can allow inferences into such relationships among traits. If the loci conditioning two traits segregate independently, it can be inferred that they are controlled by different genes. If two traits such as fitness and virulence cannot be separated by recombination, it can be concluded that they may be controlled by the same chromosomal segment and possibly by the same gene. Using this approach, an

†Corresponding author: S. Mideros; E-mail: smideros@illinois.edu

*The $\boldsymbol{e}$-Xtra logo stands for "electronic extra" and indicates that one supplementary file, eight supplementary figures, and seven supplementary tables are published online.

(C) 2018 The American Phytopathological Society association has been postulated for fungicide sensitivity and melanization genes in fungi (Lendenmann et al. 2015).

Setosphaeria turcica (Exserohilum turcicum) is a widespread hemibiotrophic pathogen of maize (Zea mays) and causal agent of northern leaf blight (NLB). Development of NLB before flowering can result in significant yield losses on susceptible hybrids, especially under moderate temperatures and high humidity (Carson 1999; Perkins and Pedersen 1987; Ullstrup and Miles 1957). Resistant hybrids are used as the main method for control of NLB. Nevertheless, NLB has consistently been one of the most damaging diseases of maize in North America in recent years (Mueller et al. 2016). This has highlighted the need for better management methods. Strategic breeding and novel control measures can be drawn from knowledge of the genetic diversity of the host-pathogen interactors (McDonald 2015), and the evolutionary forces affecting the pathogen population. For the maize-S. turcica pathosystem, these interactors are only beginning to be identified (Wu and Turgeon 2013).

As in other pathosystems involving biotrophic or hemibiotrophic pathogens, S. turcica diversity can be classified into races or pathotypes that are defined by the differential ability to overcome major $R$ genes. The $R$ genes used for race differentiation include $\mathrm{Ht1}, \mathrm{Ht} 2, \mathrm{Ht} 3$, and $\mathrm{HtN}$ (Leonard et al. 1989). A strain that is able to cause disease on maize with the $H t 1$ gene (i.e., is "compatible" with $H t l$ ) but not on plants with $H t 2$, $H t 3$, and $H t N$ is called "race 1", whereas a strain that is compatible with the Ht2, Ht3, and HtN genes but incompatible with the Htl gene is called "race 23N" (Leonard et al. 1989). This implies that race 1 lacks avirulence $(A V R)$ gene AVRHt1 but carries AVRHt2, AVRHt3, and $A V R H t N$, whereas race $23 \mathrm{~N}$ carries $A V R H t 1$ but lacks $A V R H t 2, A V R H t 3$, and $A V R H t N$. AVR genes in general are now known to be involved in virulence and are a subset of the effector repertoire that modulates the interaction between the fungus and its host (Lo Presti et al. 2015).

The frequency of race 1 isolates of $S$. turcica increased from 2 to $31 \%$ from 1974 to 1994 , presumably due to widespread cultivation of maize with the Htl gene (Ferguson and Carson 2007). The identification of genetically diverse race 1 strains only after years of widespread deployment of maize varieties carrying $H t l$ was taken as 
indirect evidence that race 1 isolates have a fitness disadvantage in the absence of plants with the Htl gene (Ferguson and Carson 2007). The consistent presence of race $23 \mathrm{~N}$ strains, even when the corresponding resistance loci had not been deployed, was taken to suggest that virulence on $H t 2, H t 3$, and $H t N$ was not associated with a fitness cost.

The gene-for-gene mechanism for the maize-S. turcica pathosystem has been known for decades (Lim et al. 1974) but little is known about the molecular identity of the $A V R$ genes or their positions in the $S$. turcica genome. The genes influencing other important fungal traits also remain to be determined. Based on the suggestion that there could be a cost of virulence on AVRHtl (Ferguson and Carson 2007), we tested the hypothesis that $A V R$ genes are genetically associated with pathogen developmental traits evaluated in vitro. We assumed that in vitro evaluated radial growth, number of conidia, and mycelial abundance are a proxy for saprophytic growth, and that saprophytic growth is a component of fitness (Akinsanmi et al. 2007; Lendenmann et al. 2015). Also, we evaluated pigmentation because field strains vary in their levels of pigmentation. In addition, certain pigments such as melanin have been associated with virulence, although this association might be due to shared pathways rather than a direct relationship (Ma et al. 2017; Xue et al. 2013; Zainudin et al. 2015).

We used a forward genetics approach to identify the regions of the $S$. turcica genome that are associated with interactions with maize genes $\mathrm{Htl}$ and $\mathrm{Ht} 2$ and also to identify the chromosomal regions associated with fitness-related developmental traits in the pathogen. To accomplish this, we created a mapping population of $S$. turcica and evaluated the resulting progeny for multiple traits in planta and in vitro. We used the resulting genetic map locations of AVRHtl, $A V R H t 2$, and loci influencing a range of fungal traits to assess the hypothesis that virulence is associated with in vitro developmental traits (as a proxy for reduced saprophytic growth and fitness) in the absence of selection by the corresponding resistance locus. We also charted scaffolds from genome sequencing onto the map. The construction of a genetic map and its alignment with the physical map is a step toward cloning of the $A V R$ genes and a better understanding of the pathogen's genetics, biology, and ecology.

\section{MATERIALS AND METHODS}

Fungal strains. Thirteen diverse field strains of $S$. turcica were used for this study (Table 1). Preliminary trials were conducted to identify candidate strains as parents of a cross that would produce progeny segregating for race. Ideally, this would be a cross between race 1 and race $23 \mathrm{~N}$, because race 1 was expected to carry functional alleles of $A V R H t 2, A V R H t 3$, and $A V R H t N$ while race $23 \mathrm{~N}$ was expected to carry AVRHt1. Only a single race 1 strain, NY001 (Chung et al. 2010), was available to us at the time of the study. Because NY001 had the MAT1-1 mating-type allele, we needed a race $23 \mathrm{~N}$ strain carrying the MAT1-2 mating type allele as the other parent. Among the test crosses between NY001 and candidate race $23 \mathrm{~N}$ strains, the cross with strain Et52B was best at producing progeny. Strains were crossed using standard protocols (Leach et al. 1982; Luttrell 1958). In brief, the two parent strains were placed on opposite sides of a stalk of Johnson grass on Sachs medium. Approximately 3 weeks after setting the cross, mature fruiting bodies were crushed and 221 single ascospores were collected under a dissecting microscope. Each recovered progeny was grown on lactose casein hydrolysate agar (LCA) plates for 10 days at room temperature and with $12 \mathrm{~h}$ of light, after which the plate was flooded with $50 \%$ glycerol solution, and conidial suspensions were transferred to cryovials for storage at $-80^{\circ} \mathrm{C}$.

Genotyping-by-sequencing analysis. A spore suspension from a 2- to 3-week-old LCA culture of each of the 234 strains (13 field strains and 221 progeny strains) was used to inoculate $50 \mathrm{ml}$ of $0.5 \times$ potato dextrose broth in a $250-\mathrm{ml}$ flask. Cultures were grown for 5 days with shaking at $120 \mathrm{rpm}$ at room temperature. Mycelium was harvested on sterile filter paper in a Büchner funnel and rinsed with sterile water. Genomic DNA was extracted from approximately 0.005 to $0.01 \mathrm{~g}$ of lyophilized mycelium following a high-throughput miniprep cetyltrimethylammonium bromide (CTAB) method (Chung et al. 2010), with the addition that the chloroform and isoamyl alcohol extraction was conducted twice. To remove polysaccharides, the airdried DNA pellet from the CTAB extraction was dissolved in $250 \mu \mathrm{l}$ of Tris-EDTA buffer (10 mM Tris- $\mathrm{HCl}$ and $1 \mathrm{mM}$ EDTA, $\mathrm{pH} 8.0)$ containing $1.5 \mathrm{M} \mathrm{NaCl}$, then centrifuged at 5,200 rpm for $5 \mathrm{~min}$. The supernatant was transferred to a new tube, mixed with 2 volumes of $100 \%$ ethanol, and incubated at $-20^{\circ} \mathrm{C}$ overnight (Fang et al. 1992). The subsequent steps (DNA precipitation, washing, air drying, and dissolving) were as previously described (Chung et al. 2010).

DNA from the 234 fungal strains was barcoded and pooled following the genotyping-by-sequencing (GBS) protocol (Elshire et al. 2011). Two DNA samples from each of strains Et34A, Et37A, Et38B, Et52B, Et54A, and NY001 were processed, giving a total of 240

TABLE 1. Characteristics of the Setosphaeria turcica field strains used in this study

\begin{tabular}{|c|c|c|c|c|c|c|c|c|c|c|c|}
\hline \multirow[b]{2}{*}{ Strain $^{b}$} & \multirow[b]{2}{*}{ Race } & \multirow[b]{2}{*}{$M A T$} & \multicolumn{7}{|c|}{ Phenotypes $^{\mathrm{a}}$} & \multirow[b]{2}{*}{$\mathrm{M}-A V R H t 2^{\mathrm{c}}$} & \multirow[b]{2}{*}{$\mathrm{M}-A V R H t 1^{\mathrm{d}}$} \\
\hline & & & $\mathrm{r} / \mathrm{s}$ & ip & dla & $\mathrm{rg}$ & pgm & abn & cnd & & \\
\hline Et26A & 0 & MAT1-2 & $\mathrm{R}$ & 17 & 1 & 31 & 2 & 1 & 119 & $\mathrm{G}$ & $\mathrm{G}$ \\
\hline Et32A & 0 & MAT1-2 & $\mathrm{R}$ & 16 & 3 & 27 & 2 & 1 & 247 & G & G \\
\hline Et37A & 0 & MAT1-2 & $\mathrm{R}$ & 14 & 10 & 49 & 2 & 2 & 98 & $\mathrm{G}$ & $\mathrm{G}$ \\
\hline Et38B & 0 & MAT1-1 & $\mathrm{R}$ & 17 & 1 & 52 & 3 & 2 & 63 & G & G \\
\hline Et39A & 0 & MAT1-1 & $\mathrm{R}$ & 16 & 3 & 32 & 2 & 1 & 77 & $\mathrm{G}$ & $\mathrm{G}$ \\
\hline Et40A & 0 & MAT1-1 & $\mathrm{R}$ & 14 & 5 & 45 & 2 & 1 & 128 & G & $\mathrm{G}$ \\
\hline Et54A & 0 & MAT1-2 & $\mathrm{R}$ & 17 & 1 & 19 & 2 & 1 & 223 & G & G \\
\hline Et62A & 23 & MAT1-2 & $\mathrm{S}$ & 10 & 30 & 30 & 2 & 1 & 102 & $\mathrm{C}$ & G \\
\hline Et63A & 23 & $M A T 1-2$ & $\mathrm{~S}$ & 10 & 50 & 47 & 3 & 2 & 164 & $\mathrm{C}$ & G \\
\hline Et34A & $-^{\mathrm{e}}$ & MAT1-2 & $\mathrm{R}$ & 16 & 5 & 28 & 2 & 1 & 414 & G & G \\
\hline Et28A & $23 \mathrm{~N}$ & MAT1-1 & $\mathrm{S}$ & 10 & 50 & 60 & 3 & 2 & 86 & G & G \\
\hline $\mathrm{Et} 52 \mathrm{~B}^{\mathrm{f}}$ & $23 \mathrm{~N}$ & MAT1-2 & $\mathrm{S}$ & 10 & 52 & 33 & 2 & 1 & 185 & $\mathrm{C}$ & $\mathrm{G}$ \\
\hline NY001 ${ }^{\mathrm{f}}$ & 1 & MAT1-1 & $\mathrm{R}$ & 13 & 18 & 47 & 3 & 3 & 78 & $\mathrm{G}$ & A \\
\hline
\end{tabular}

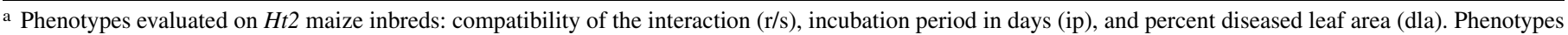
evaluated on axenic media: radial growth at 13 days after inoculation, in millimeters (rg), pigmentation on a scale of 1 to 3 (pgm), mycelial abundance on a scale of 1 to $3(\mathrm{abn})$, and number of conidia per count (conidiation = cnd). Note that the field strains (except for the parents of the population) were evaluated only once; therefore, no statistical inference is intended.

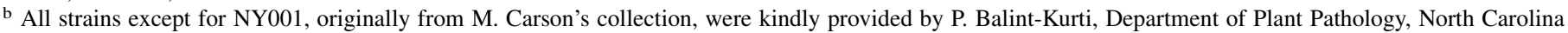
State University. NY001 was isolated from an infected leaf sample kindly provided by G. Bergstrom, Section of Plant Pathology and Plant-Microbe Biology, Cornell University.

c Genetic mapping indicated that the most significantly associated marker to AVRHt2 was S8_2099705.

d Genetic mapping indicated that the most significantly associated marker to AVRHt1 was S2_3552054.

e Strain Et34A was originally characterized as race $23 \mathrm{~N}$ but our phenotypes and genotypes indicated that it is not a race 2 strain.

f Used as parental strains in this study. 
samples. Briefly, for each library, DNA samples were digested with the restriction enzyme ApeKI, ligated to 48 barcode adapters, and amplified by polymerase chain reaction (PCR). Sequencing was conducted using an Illumina Genome Analyzer II (Illumina, San Diego, CA) at Cornell University's Biotechnology Resource Center (http://www.biotech. cornell.edu/biotechnology-resource-center-brc). Five lanes of sequences were generated.

To confirm the robustness of our genotypes, we used six pipelines for calling single-nucleotide polymorphisms (SNP). For the primary pipeline, unfiltered Illumina data were assigned to individual samples using the barcode sequence and trimmed to $64 \mathrm{bp}$ using TASSEL 3.0 (Elshire et al. 2011). Only sequences that had an exact match to a barcode and the restriction enzyme overhang were retained. To control for possible sequencing errors, sequence reads were used only if they were represented at least five times. For the reference genome, we used the $S$. turcica genome sequence for race $23 \mathrm{~N}$, MAT1-1 strain 28A (Et28A-v1.0), which consisted of $43 \mathrm{Mb}$ on 407 scaffolds with a scaffold L50 of $2.14 \mathrm{Mb}$ (https://genome.jgi.doe.gov/Settu1/Settu1. home.html) (Condon et al. 2013; Ohm et al. 2012). Filtered reads were aligned to Et28A-v1.0 using the Burrows-Wheeler 0.6.1 alignment software, with default parameters (Li and Durbin 2009). SNP were called by creating alignments for each set of sequence tags that started at the same genomic position using TASSEL 3.0 (Bradbury et al. 2007). The minimum value for inbreeding coefficient was set at 0.05 , a minimum minor allele frequency (MAF) of $1 \%$, minimum minor allele counts of 10 , minimum locus coverage of $10 \%$, and rare alleles as well as gaps were ignored.

We first created a set of SNP for the 13 field strains by filtering for a minimum of $10 \%$ coverage. We then consolidated the SNP calls for the six strains that were genotyped in duplicate into a single set of sequence variants. This set, henceforth termed the "diverse set", contained 9,359 SNP that had a polymorphism between at least 2 of the 13 field strains. We created a set of SNP for the progeny, starting with the diverse set and filtering out the heterozygous calls. We kept variants with at least 22 calls for each SNP ( $10 \%$ of the population), and $20 \%$ minimum MAF. We also kept SNP for which we had information from at least one of the parents and discarded 109 SNP that had nonparental genotypes. This set of variants was termed the "population set" and contained 2,078 SNP.

Secondary genotypic pipelines. To confirm the robustness of the primary SNP-calling pipeline, we used five secondary pipelines to call SNP in the NY001 $\times$ 52B progeny: the TASSEL 5.0 GBSv2 pipeline (Glaubitz et al. 2014), two pipelines using GATK (DePristo et al. 2011), and two pipelines using Freebayes (Garrison and Marth 2012). In all cases, those reads that did not pass automatic Illumina filtering criteria were filtered out.

In the TASSEL GBSv2 pipeline, reads were trimmed and filtered for a Q-score of 20 throughout the entire trimmed read. Filtered reads were aligned to the Et28A-v1.0 genome sequence with bowtie2 2.2.6 (Langmead and Salzberg 2012) using very sensitive local alignment default parameters. SNP were called from these alignments with a minimum MAF of 0.01 , minimum locus coverage of 0.1 , and assumed error rate of 0.01 , but with no cutoff for the inbreeding coefficient

In the GATK and Freebayes pipelines, reads were trimmed and filtered using process_radtags from the Stacks pipeline (Catchen et al. 2013). Reads with an average quality score $<10$ over a sliding window of $10 \mathrm{bp}$ were discarded. Alignment was conducted with bowtie 2 as indicated above, and aligned reads were sorted with bamtools (Barnett et al. 2011) and processed with bamaddrg. SNP were called with Freebayes or GATK under default settings, with ploidy set for haploid and diploid. SNP were filtered with bcftools using a Q-score cutoff of 40.

For all secondary pipelines, SNP calls were then filtered as described for the main pipeline, keeping SNP present in at least $10 \%$ of the population, with a minimum MAF of $20 \%$, and for which there was genetic information from at least one parent. The resultant SNP calls were collectively termed the "secondary population sets".

Genetic map. The population set of variants was converted to R/QTL format using a custom Perl script, while the secondary population sets were converted using a custom R script. For both sets, further filtering was conducted in R/QTL by retaining markers that had data for at least 20 strains, dropping markers with identical genotypes, and dropping markers showing segregation distortion. Markers formed linkage groups (LG) if they had a maximum recombination fraction of 0.45 and a minimum logarithm of odds (LOD) of 6. Marker order was assigned for each LG by iteratively changing marker positions in a sliding window to produce the most likely order using the ripple function in R/QTL. Marker order was also informed by its position in Et28A-v1.0. With a draft version of the genetic map assembled, further quality control was conducted by discarding one marker at the time, counting cross overs, and calculating genotyping error LOD scores (Broman and Sen 2009). The five secondary sets of variants were also used to construct secondary genetic maps to confirm the robustness of the above genetic map and to see if any could make a more complete map. These maps were constructed as described above for the primary genetic map.

Phenotypes. In planta traits were evaluated to identify the location of the $A V R$ genes. Virulence on $H t 2$ maize was assessed by inoculating near-isogenic lines (NIL) containing the Ht2 ${ }_{D K 888}$ or the $h t 2_{S 11}$ alleles (Chung et al. 2010). The experiment was arranged as an incomplete block design, where the experimental plot was composed of five plants of each maize NIL (10 plants total). The greenhouse contained eight blocks, and each block had nine experimental plots. Each plant was inoculated with one of the fungal strains (randomly assigned) by pipetting $0.5 \mathrm{ml}$ of spore suspension $(4,000$ conidia $/ \mathrm{ml}$ in $0.02 \%$ Tween 20 ) into the whorl. Two plots in each block were inoculated with the parents of the population. Because not all treatments could fit into a single greenhouse, the experiment was divided into four subsets and inoculated at four times from May to June 2011. Greenhouse conditions were set at 22 and $18^{\circ} \mathrm{C}$ day and night temperatures, respectively. Plants were inoculated 19 to 25 days after planting (four leaf collars visible: stage V4) and kept at $100 \%$ humidity for $24 \mathrm{~h}$ using a custom-built moist chamber that covered each bench in the greenhouse. Incubation period (IP) was determined on an individual plant basis by observing the plants twice a day and noting the number of days after inoculation when lesions were first observed. After all of the plants showed lesions (13 to 18 days postinoculation[dpi]), two infected leaves per plant were carefully collected and photographed under the same conditions on the collection day. The images were used for disease evaluation. Compatibility with $\mathrm{Ht} 2$ was visually rated from the photographs by assigning a " 1 " for a resistant interaction and a " 0 " for a susceptible interaction. The resistant reaction for $\mathrm{Ht} 2$ was characterized by chlorotic smaller lesions on $\mathrm{Ht} 2$ plants compared with necrotic larger lesions on $h t 2$ plants, while the susceptible reaction for $H t 2$ was characterized by expanding necrotic lesions on both $H t 2$ and $h t 2$ NIL (Supplementary Fig. S1). In a few cases, a score of 0.5 was assigned if the differences between the NIL were not clear. Diseased leaf area (DLA) was visually rated from the pictures of the five plants on each subplot as a percentage of infection.

The interaction on maize containing the $H t l$ gene was evaluated by inoculating maize line Pa91 Htl. The experiment was arranged in a complete block design. Each experimental plot was composed of three plants in a single $15-\mathrm{cm}$-pot. The experiment contained 454 plots. Two plots were inoculated at V4 with each progeny by pipetting $0.5 \mathrm{ml}(4,000$ conidia $/ \mathrm{ml}$ in $0.02 \%$ Tween 20$)$ of spore suspension into the whorl. In addition, 20 plots were inoculated with the parental strains. Each replicate was placed on different benches in the same greenhouse and maintained at $100 \%$ humidity for $24 \mathrm{~h}$ using a custom-built humidity chamber adapted to each bench. Greenhouse conditions were the same as indicated above and the experiment was conducted during April and May 2013. Compatibility with HtI was evaluated $10 \mathrm{dpi}$ and scored as susceptible or resistant, then transformed to 0 or 1 for data analysis.

Several fungal traits with potential relevance to fitness were evaluated in vitro. Four LCA plates were inoculated with a 5- $\mu \mathrm{l}$ spore suspension (4,000 conidia/ml; approximately 20 spores), and cultures were grown in a controlled temperature incubator at 
$25^{\circ} \mathrm{C}$, with a 12 -h light-and-dark cycle. Plates were arranged in a randomized complete block design, with the parental strains repeated in every block. Mycelium abundance was rated on 6-day-old plates on a scale of 1 to 3 , where the highest number was assigned to strains with the most profuse aerial mycelium (Supplementary Fig. S2). Radial growth was measured by determining the diameter of each colony using a ruler at 6 and 13 dpi. Pigmentation was visually rated at 6 dpi using the following scale: $1=$ strains with little pigmentation, $2=$ strains with some diffuse pigment in the center, and $3=$ distinct dark pigmentation beneath the colony. Finally, to determine asexual sporulation (conidiation), four discs $6.35 \mathrm{~mm}$ in diameter were cut from the middle part of each 13-day-old colony using a sterilized drinking straw. The discs were transferred to a microtube containing $1 \mathrm{ml}$ of double-distilled $\mathrm{H}_{2} \mathrm{O}$ with $0.02 \%$ Tween 20 . After vortexing, the number of spores in four 5- $\mu$ l droplets was counted under a dissecting microscope, with the aid of a transparency film with a grid printed on it. Preliminary data showed that this method was as accurate as counting with an hemocytometer and allowed higher throughput.

Phenotypic data analysis. As indicated above, IP and DLA data were collected from the two NIL ( $H t 2$ and $h t 2$ ). We analyzed the raw data and, in addition, created a compound phenotype for each experimental plot by subtracting IP on $h t 2$ from IP on Ht2 (DIP; resistant interactions had higher value) and by subtracting DLA on $H t 2$ from DLA on $h t 2$ (DDLA; resistant interactions had higher value). All phenotypes were then analyzed using the lme 4 package version 1.1-10 (Bates et al. 2015) on $\mathrm{R}$ version 3.2.2 (R Core Team 2015). Preliminary data analysis indicated that repeated counts from each tube for asexual sporulation and experiment subsets for the compatibility with $\mathrm{Ht} 2$ were not sources of significant variation and, therefore, were not included as factors on further statistical analysis. For colony pigmentation and compatibility with $H t 2$, the parental controls did not vary from block to block. For all phenotypes except for pigmentation and compatibility with $\mathrm{Ht2}$, best linear unbiased estimators (BLUE) were computed using the following model: $Y_{i j}=S_{i}+B_{j}+E_{i j}$, where $S_{i}$ is the fixed effect of the $i$ th strain, $B_{j}$ is the random effect of the $j$ th complete or incomplete block (depending on the phenotype), and $E_{i j}$ is the random residual error effect for the $i$ th strain in the jth block. Quantile-quantile plots and the ShapiroWilk test were applied to all phenotypes as well as natural log and square root transformations.

Quantitative trait loci mapping. Quantitative trait loci (QTL) were mapped using the R/QTL package (Broman and Sen 2009) in $\mathrm{R}$ version 3.2.2 ( $\mathrm{R}$ Core Team 2015) using estimated BLUE or raw data for pigmentation and compatibility with $\mathrm{Ht} 2$ and the population set of markers. The multiple imputation method with single QTL models (Sen and Churchill 2001) was used for all traits, and 8,192 draws were produced at 1-centimorgan (cM) steps. In addition, nonparametric QTL mapping was applied to pigmentation data, and the binary QTL mapping method was applied for compatibility with $H t 2$. We used 1,000 permutations to identify significance thresholds for each trait. Estimates of the location of each QTL were calculated using 1.5 LOD support and Bayes credible estimates. The effect of each QTL (the result of the subtraction of the average phenotype for the Et52B allele from the NY001 allele) was estimated using the effectplot function for the most significant marker or pseudomarker. Percent variation for single QTL was calculated using the makeqtl and fitqtl functions in R/qtl. In addition, for the compatibility with $\mathrm{Htl}$, marker regression was conducted using the scanone function. To visualize the colocation of $A V R$ genes with fitness-related QTL, a figure was created, in part, using the ggplot 2 package on $\mathrm{R}$, with minor changes to the code first reported by Lovell et al. (2015).

Analysis of $\boldsymbol{A} \boldsymbol{V} \boldsymbol{R}$ regions. To identify molecular markers associated with the ability to cause disease on plants with the Htl and $H t 2$ genes, we examined the regions identified by QTL mapping. For the AVRHt2 region, we first identified the closest molecular markers outside of the most conservative confidence intervals for the plantassociated traits. For the AVRHt1 region, only single-marker regression produced a significant candidate gene location; therefore, we chose an arbitrary region of the approximate same physical map size as that identified for the AVRHt2 region at approximately marker S2_3552054. Then, we identified all GBS markers in the population set in both regions $(n=54)$ of scaffolds $2,8,19$, and 23 , and conducted a multiple regression analysis on those markers using only the binary compatibility phenotypes on $\mathrm{Ht} 2$ or $\mathrm{Ht} 1$ plants.

Primers for an SNP-specific assay were designed for a marker not covered by the GBS procedure (S2_3549698) using Primer 3 (Untergasser et al. 2012). The variant specific primers (S2_3549698_C GAAGGTGACCAAGTTCATGCTCGATGAATACTATGCCAA TCTCC, S2_3549698_T GAAGGTCGGAGTCAACGGATTCGAT GAATACTATGCCAATCTCT, and S2_3549698R CCCTTGCT CTAGACCCCAT) contained the 6-FAM- and VIC-specific tags for a kompetitive allele-specific PCR (KASP) assay (LGC, Middlesex, $\mathrm{UK})$. PCR of all the progeny and the diverse strains was conducted in a 384-well plate that contained $2 \mu \mathrm{l}$ of DNA, $2 \mu \mathrm{l}$ of KASP Master mix (LGC), $0.055 \mu \mathrm{l}$ of primer mix (12 $\mu$ l of each allele-specific primer at $100 \mu \mathrm{M}, 30 \mu \mathrm{l}$ of the reverse primer at $100 \mu \mathrm{M}$, and $46 \mu \mathrm{l}$ of water), and $0.032 \mu \mathrm{l}$ of $\mathrm{MgCl}_{2}(50 \mathrm{nM})$. The assay was repeated three times.

\section{RESULTS}

Molecular markers. Sequencing yielded 142,644,194 reads with an average of 28,528,839 reads per lane (PRJNA329527). Quality control and collapsing of repeated reads produced 483,779 tags (identical consensus sequences found in one or more libraries) were retained. Of these, 462,081 aligned to Et28A-v1.0, resulting in a total coverage of $0.72 \times$ and an average coverage of $0.003 \times$ for each of the strains. The SNP-calling algorithm produced 27,174 SNP across 171 Et28A-v1.0 scaffolds (out of 407), for an average coverage of $0.63 \mathrm{SNP} / \mathrm{kb}$. For this set, $35 \%$ of the SNP calls were missing and $3.7 \%$ were called heterozygous. SNP naming reflected their position on Et28A-v1.0. For example, S8_2099705 aligned to scaffold 8, position 20999705. Further filtering to produce the diverse set of markers resulted in 9,359 SNP, with $31 \%$ missing calls and $7 \%$ heterozygous calls. The population set of markers had $2,078 \mathrm{SNP}$, with $65 \%$ missing calls and no heterozygous calls.

Most of the secondary SNP-calling pipelines performed similar to or worse than the primary pipeline. Except for the TASSEL GBSv2 pipeline, all produced fewer markers than the primary pipeline, though with lower missing data (Supplementary Table S1). In all cases, the average MAF was approximately 0.35 , indicating an overall trend of segregation distortion. All nonhaploid pipelines had a fairly high number of heterozygous calls, between 8.7 and $23 \%$ of all SNP genotypes. These two trends were driven by a large group of SNP with a high number of heterozygous calls and highly distorted segregation, as discussed below.

The SNP called by the different pipelines were somewhat inconsistent but with a set of core markers. Any two SNP-calling programs shared between 398 and 816 markers (16 to 59\% of markers), with diploid and haploid versions of the same program having mostly the same markers (Supplementary Table S2). All pipelines shared a core set of 271 markers. Of these 271 markers, 182 (67\%) were in the primary genetic map.

All marker sets contained only a few thousand markers, despite having been derived from 142 million reads. This was primarily due to the low number of polymorphisms between 52B and NY001. Only $15 \%$ of reads were filtered out because of low quality, errors in the barcode or restriction site, or lack of alignment to the $S$. turcica genome (Supplementary Table S3). Between 50 and $90 \%$ of reads were filtered out because the assembled GBS tag contained no polymorphisms (not even errors), while 72 to $92 \%$ of reads were filtered out because the MAF was $<0.2$, indicative of segregation distortion or sequencing errors (Supplementary Table S4). Thus, the final SNP calls were made with only 1.2 to 3.2 million reads.

Heterozygous genotypes. In all of the nonhaploid SNP-calling pipelines, a high proportion of the SNP calls were heterozygous. 
The rate of heterozygosity ranged from $8.7 \%$ in the primary pipeline (before heterozygous SNP calls were removed) to 23 in the secondary TASSEL GBSv2 pipeline. Regardless of the pipeline used, SNP fell into two distinct groups. One group had a low proportion of heterozygous calls $(\leq 5 \%$ of the NY001 $\times 52 \mathrm{~B}$ population called as heterozygous) and a MAF close to 0.5 (i.e., 1:1 segregation) (Fig. 1, lower group in each panel). The other group had a high rate of heterozygous calls ( $>5 \%$ of the NY001 $\times 52 \mathrm{~B}$ population called as heterozygous) and a MAF ranging from 0.2 to 0.5 (i.e., variable and often distorted segregation) (Fig. 1, upper group in each panel). Thus, the low-heterozygosity SNP looked as expected for markers in a haploid species (Knox-Davies and Dickson 1960), while the high-heterozygosity SNP did not. As discussed below, these groups may represent singlecopy and multicopy regions of the $S$. turcica genome, respectively.

This was consistent with the depth of GBS reads at the different SNP locations. In the three secondary SNP-calling pipelines in which there were heterozygous calls, the median depth of GBS reads was roughly three times higher at high-heterozygosity SNP than at lowheterozygosity SNP positions, as would be expected if the latter represented multicopy sites (Supplementary Fig. S3). For all three pipelines, the mean log-transformed read depths for the two groups of SNP were significantly different, as determined by $F$ test $(P<2 \mathrm{e}-16)$.

Genetic map. After discarding markers with data for $<20$ strains $(n=773)$, markers with identical genotypes $(n=155)$, and markers with segregation distortion $(n=153)$, a first linkage map was produced. Other markers $(n=625)$ did not form an LG under the specified parameters. Further quality control, conducted by dropping one marker at the time, identifying markers with high likelihood of being genotyping errors, and another round of order comparison in a sliding window using the ripple function indicated that 70 markers were not properly located. All of these had over $50 \%$ missing genotypes and were deleted from the genetic map.
The mean and median number of crossovers per individual was 15. One individual (St136x) with an unexpectedly high number of 33 crossovers was discarded. Our preliminary calculations indicated that a progeny size of 150 was sufficient for $10-\mathrm{cM}$ resolution, insensitive to missing values as high as $20 \%$ and typing errors of $3 \%$. The final genetic map, composed of 302 markers and $21 \mathrm{LG}$, spanned 1,981 cM, with an average space between markers of $7.1 \mathrm{cM}$ and maximum spacing of $39.4 \mathrm{cM}$ (Fig. 2; Supplementary Table S5). The LG were given designations (LG 1, 2...21) in order of decreasing size.

None of the alternative SNP-calling pipelines produced a more complete or marker-rich genetic map. The secondary genetic maps were generally the same size in centimorgans as the primary genetic map but all had fewer markers (Supplementary Table S6). Although the GBSv2 population set of markers was larger than the primary population set, the resultant GBSv2 genetic map was only 1,675 cM. The other maps were all roughly $2,000 \mathrm{cM}$ in length, comparable with the primary map. All maps had roughly $20 \mathrm{LG}$. There were 119 markers shared by all maps, with any given pair of maps sharing between 149 and 222 markers (Supplementary Table S7).

SNP with a high rate of heterozygous calls (in $>5 \%$ of the NY001 $\times 52 \mathrm{~B}$ population), which often had distorted segregation (Fig. 1, upper group in each panel), could generally not be placed in genetic maps. Thus, all of the genetic maps were mostly made from SNP with a low rate of heterozygous calls (in $\leq 5 \%$ of the NY001 $\times$ 52B population), which tended to have 1:1 segregation, as expected (Fig. 1, lower group in each panel). This was true for the maps made from the secondary SNP-call pipelines as well.

Location of $\boldsymbol{A V R H t 2}$ and $\boldsymbol{A V R H t 1}$. Based on multiple imputation QTL mapping of the traits rated in planta for the interaction with $\mathrm{Ht2}$, the AVRHt2 locus mapped to LG 4 (Fig. 3). The confidence intervals overlapped for the three traits measured (compatibility, difference

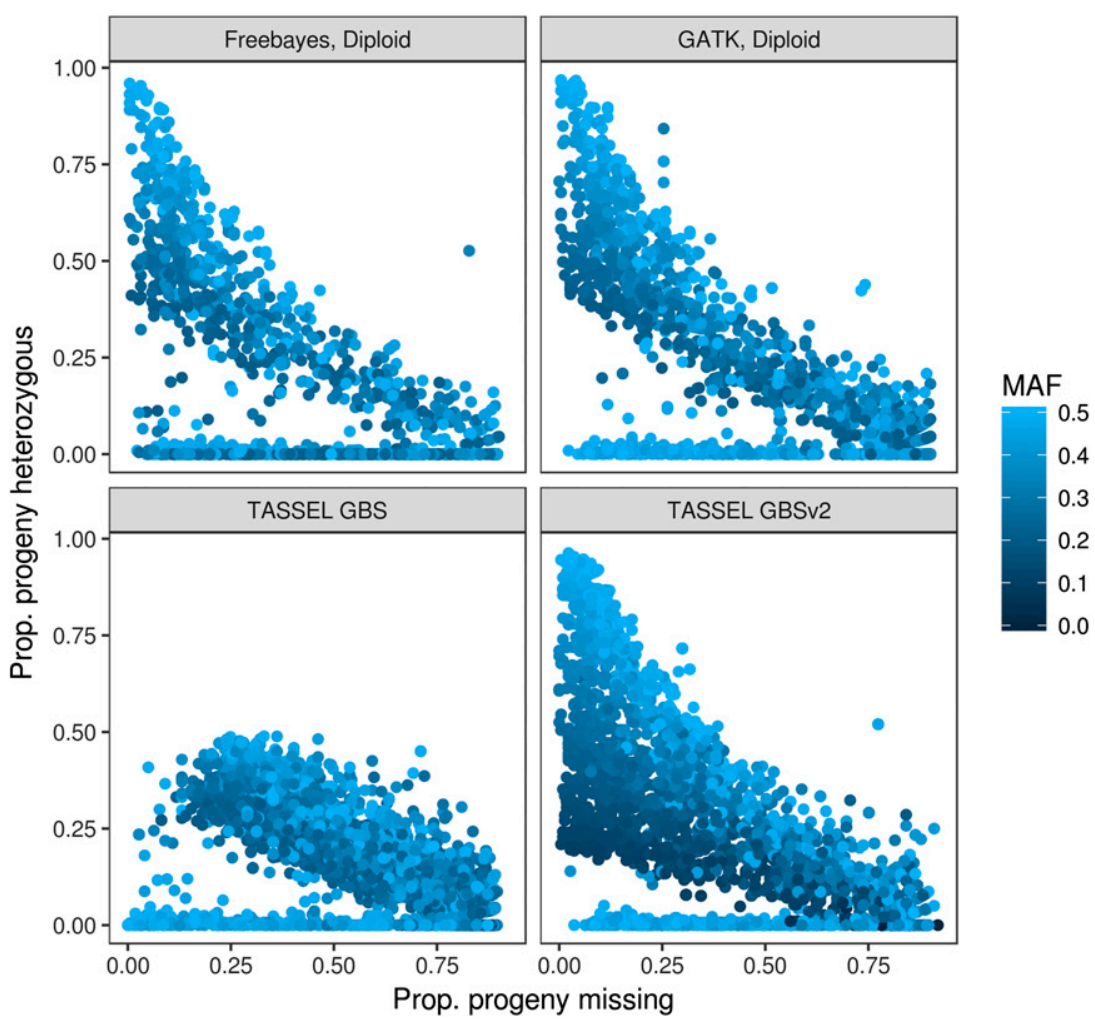

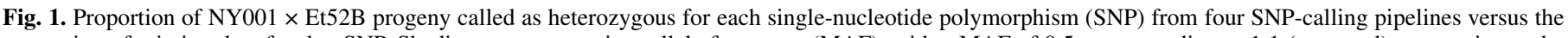

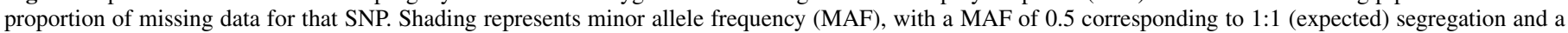

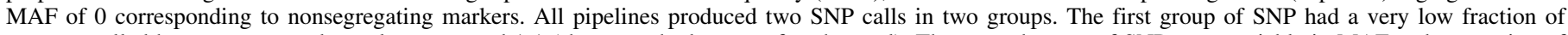

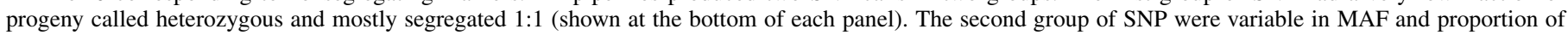

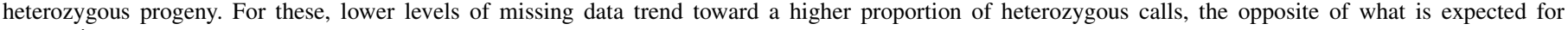
genotyping errors. 
in incubation period, and difference in diseased leaf area). Compatibility of the interaction with $\mathrm{Ht} 2$ had the smallest confidence interval: $3 \mathrm{cM}$ (Table 2). The most significant marker for the region likely containing the $A V R H t 2$ gene was marker S8_2099705. At this marker, progeny with the race 1 nucleotide produced a resistant interaction on plants with the Ht2 gene, as reflected by the differences in compatibility rating, incubation period, and diseased leaf area (Table 2). Markers S8_2099705 and S23_108733 flanked the confidence interval. Single-marker regression using the population set (expanded number of markers) also indicated that S8_2099705 was the most significant marker (LOD > 16). Significant levels of association were observed for several markers on scaffold 23, with the most significant marker being S23_102252 $(\mathrm{LOD}=5.8)$. For marker S8_2099705, most strains that produced a resistant interaction on plants with the $H t 2$ gene contained the race 1 allele (nucleotide $\mathrm{G}$ ) and most strains that produced a susceptible interaction contained the race $23 \mathrm{~N}$ allele (nucleotide $\mathrm{C}$ ) (Table 3). For the field strains of known race, all of the race 0 and race 1 strains had the race 1 allele (Table 1). Three race 2 strains (Et52B, Et62A, and Et63A) (Table 1) had the expected race 2 allele but the race $23 \mathrm{~N}$ strain Et28A did not have the nucleotide expected for race 2.

Based on single marker regression of the ability of the progeny to cause disease on Pa91Ht1 plants, AVRHt1 was associated with SNP S2_3552054 (LOD > 25). This association remained significant using multiple methods and controlling for multiple tests with variable sets of markers (data not shown). During the creation of the genetic map, marker S2_3552054 was initially linked with SNP that aligned to scaffold 6 on LG 18. However, the map positions among markers from scaffold 6 and marker S2_3552054 were separated by a gap of 230 cM. Forcing marker S2_3552054 into LG 1 (with other markers that aligned to scaffold 2) produced a gap of 1,001 cM. Therefore, marker S2_3552054 was excluded from the genetic map.
Without marker S2_3552054 in the dataset, there was no significant evidence of a QTL for the ability of the progeny to cause disease on Pa91Ht1 plants.

Using the population set of markers in the AVRHt1 region, LOD values decayed rapidly around marker S2_3552054 (LOD = 10.1). The closest markers (by physical position on Et28A-v1.0), S2_3491272 and S2_3601995, had LOD of 4.7 and 0.2, respectively. Analysis of Et28A-v1.0 indicated that S2_3552054 caused a synonymous $\mathrm{C}$ to A polymorphism in a $12-\mathrm{kb}$ gene encoding a predicted hybrid polyketide synthase (PKS) nonribosomal synthetase (NRPS): Joint Genome Institute (JGI) protein ID 179218. This gene was already a candidate virulence determinant (Wu et al. 2013). Closer examination of the GBS data indicated that the A nucleotide was present only in strain NY001 and not in any of the field strains genotyped in this study (Table 1). The closest nonsynonymous polymorphism between NY001 and the Et28A-v1.0 was identified in the same gene at position 3549698 on scaffold 2. This region was not sequenced by the GBS procedure. SNP-specific KASP assays for marker S2_3549698 segregated perfectly with the race 1 phenotype in 199 progeny strains (Table 3). The SNP-specific PCR assay also indicated the presence of strains heterozygous for SNP S2_3549698 in numbers similar to strains carrying the NY001 and the Et52B alleles. Heterozygous calls for both markers always produced resistant interactions (Table 3). Pairwise linkage between S2_3549698 and S2_3552054 was significant $\left(\mathrm{D}^{\prime}=0.97, r^{2}=\right.$ $0.83, \chi^{2} P$ value $\left.<0.0001\right)$

There were no QTL identified for any of the in planta traits on the $h t 2_{S 11}$ isoline (which did not carry any $R$ genes). The DLA and IP on $h t 2_{S 11}$ were not significantly different between lines that did or did not induce a resistance reaction on Pa91Htl $(P=0.46$ and 0.28 , respectively) or between lines that did or did not induce a resistance reaction on $H t 2_{D K 888}(P=0.25$ and 0.095 , respectively), as determined by $F$ test. When the presence or absence of AVRHt1 and AVRHt2

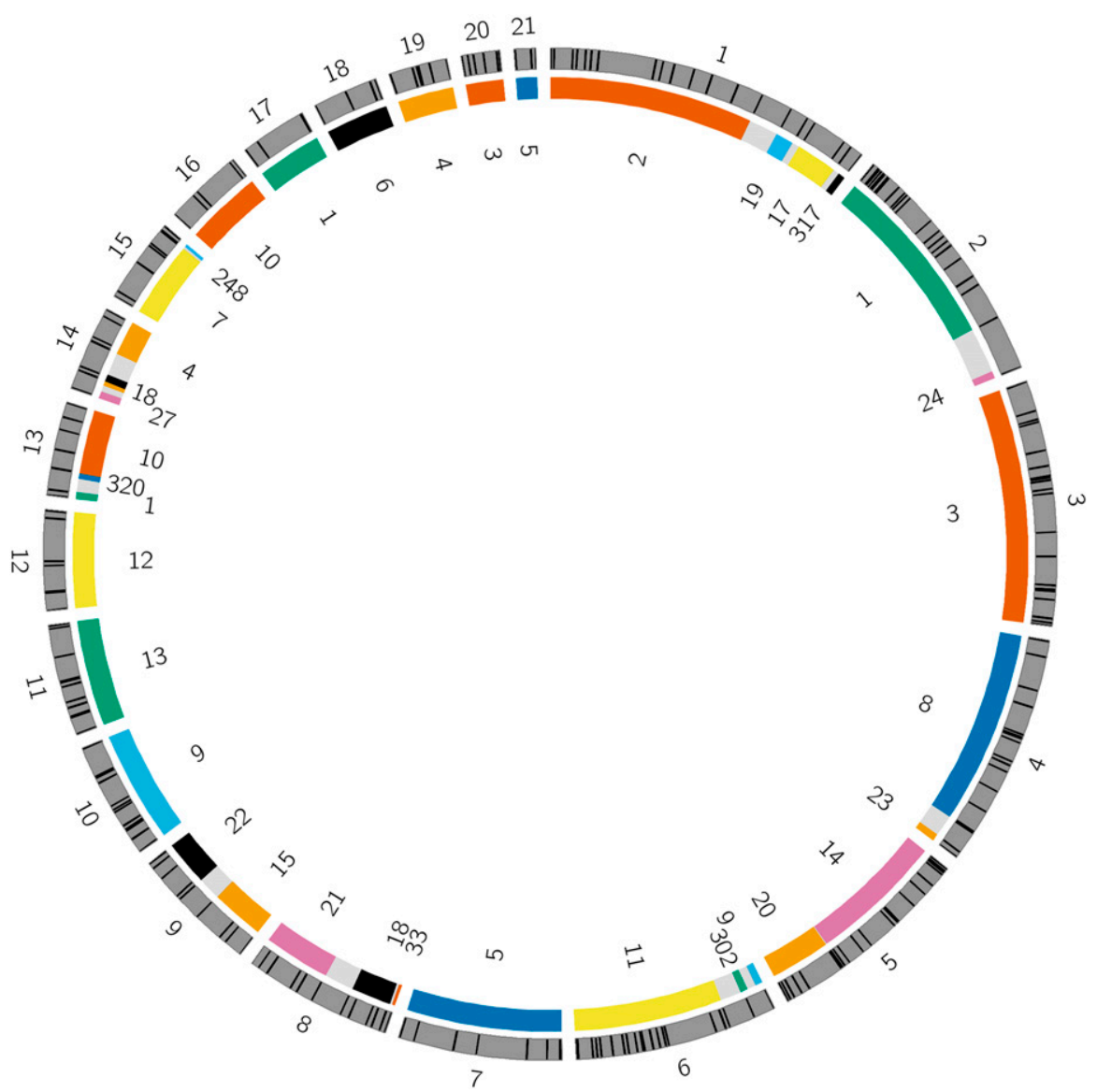

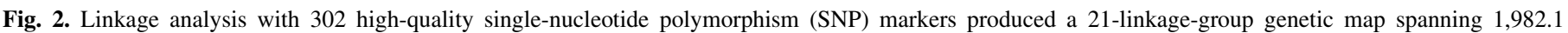

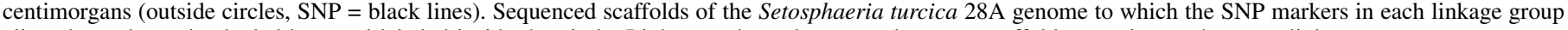
aligned are shown in shaded bars and labeled inside the circle. Light gray bars show gaps between scaffolds mapping to the same linkage group. 
isolates were considered together, however, there was a suggestive relationship between the total number of wild-type $A V R$ genes in each isolate and the DLA on $h t 2_{S 11}$. Lines with both wild-type $A V R$ genes had a higher DLA on the host with no $R$ gene $(P=0.056)$ (Fig. 4).

Location and effect of fungal phenotypes assessed in vitro. Four quantitative traits were evaluated in the progeny in vitro. Although histograms for all traits resembled normal distributions, the quantile-quantile plots and Shapiro-Wilk test suggested that only radial growth at $6 \mathrm{dpi}$ had a normal distribution. Natural log and square root transformations resulted in a normal distribution only for the conidiation data. Because QTL mapping for transformed and nontransformed data produced very similar values, only results from nontransformed data are reported. Permutation analyses with an $\alpha=$ 0.05 resulted in LOD thresholds for each trait that ranged from 2.61 to 2.87. The extent of the confidence interval using the $1.5 \mathrm{LOD}$ threshold resulted in larger (more conservative) estimates of the location of the QTL than Bayes credible estimates. Bayes credible estimates are not shown. There was significant evidence for the presence of QTL on LG 1, 2, 4, and 18 (Table 2; Fig. 3).

The QTL on LG 1 was significant for radial growth at 13 dpi. Progeny carrying the race $23 \mathrm{~N}$ (strain Et52B) allele were, on average, $5.2 \mathrm{~mm}$ larger than those with the race 1 (strain NY001) allele. QTL on LG 2 were significant for pigmentation and mycelial abundance and had overlapping interval estimates with closely located significance peaks. Progeny with the race 1 allele were almost one point higher in pigmentation rating and half a point lower in mycelial abundance than those with the race $23 \mathrm{~N}$ allele (both were assessed using three-point scales).

The QTL on LG 4 was significant for mycelial abundance and radial growth. The allelic effects for mycelial abundance and radial growth were opposite from those QTL found in LG 1 and LG 2, with progeny containing the race 1 (strain NY001) allele producing larger colonies with more abundant mycelium than those with the race $23 \mathrm{~N}$ (strain Et52B) allele. The QTL on LG 4 overlapped with the three in planta traits used to map AVRHt2 between map positions 93 and 163.8

TABLE 3. Phenotypes for the allelic variants of the most significant markers among the NY001 $\times$ Et52B progeny strains and the diverse set of markers ${ }^{\mathrm{a}}$

\begin{tabular}{llcccc}
\hline Locus & SNP & Nucleotide & Allele source & Resistant & Susceptible \\
\hline AVRHt2 & S8_2099705 & G & Race 1 & 118 & 4 \\
AVRHt2 & S8_2099705 & C & Race 23N & 6 & 73 \\
AVRHt1 & S2_3549698 & T & Race 1 & 0 & 61 \\
AVRHt1 & S2_3549698 & C & Race 23N & 64 & 0 \\
AVRHt1 & S2_3549698 & T/C & - & 61 & 0 \\
AVRHt1 & S2_3552054 & A & Race 1 & 12 & 58 \\
AVRHt1 & S2_3552054 & G & Race 23N & 43 & 0 \\
AVRHt1 & S2_3552054 & A/G & - & 77 & 0
\end{tabular}

a Compatibility phenotypes were rated as resistant or susceptible. For AVRHt2, the phenotype was evaluated on plants carrying the $H t 2_{D K 888}$ or $H t 2_{S 11}$ alleles whereas, for AVRHt1, phenotypes were evaluated on the Pa91Ht1 maize line. S2_3549698 was an exception: it was genotyped with a single-nucleotide polymorphism (SNP)-specific PCR assay.
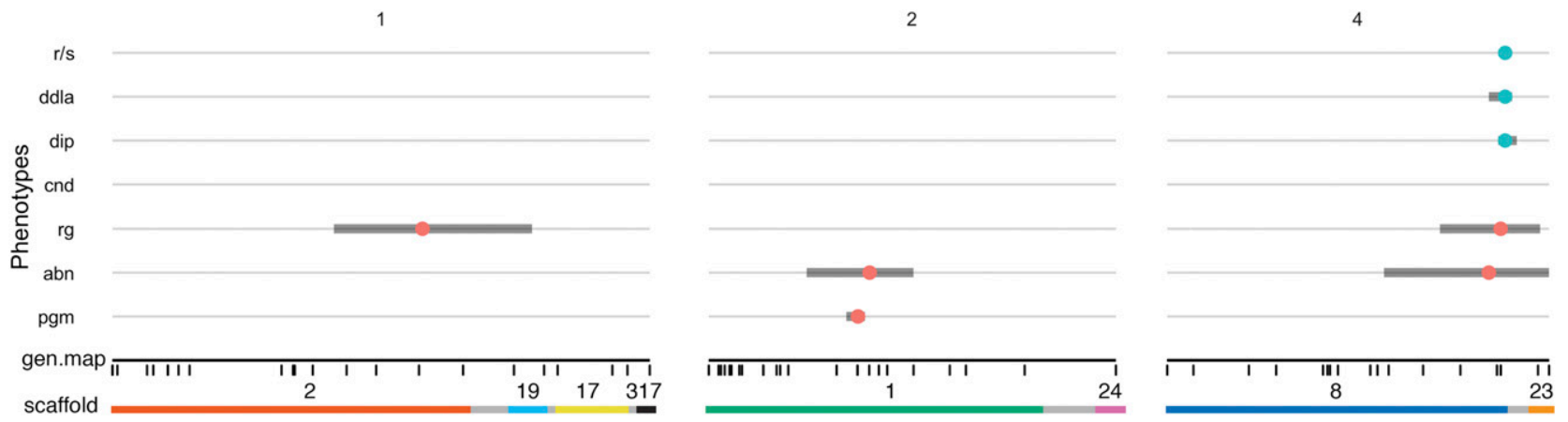

Fig. 3. In planta virulence traits (top three rows) and in vitro fungal development traits (rows four to seven) mapped to linkage groups 1,2 , 4, and 18 . Confidence intervals (1.5 logarithm of odds) are shown as gray bars. To map the location of avirulence (AVR) gene AVRHt2, maize seedlings with and without the $H t 2$ gene were inoculated with NY001 $\times$ Et52B progeny and rated for the compatibility of the interaction $(\mathrm{r} / \mathrm{s})$, diseased leaf area difference (ddla), and difference in infection period (dip). To map the location of developmental traits, progeny were evaluated in vitro and rated for conidiation (cnd), radial growth (rg), mycelial abundance (abn), and pigmentation (pgm). Scaffolds of the reference Setosphaeria turcica (Et28A-v1.0) genome to which the molecular markers are anchored are shown on the bottom row. AVRHt2 mapped to linkage group 4 colocalizing with quantitative trait loci (QTL) for radial growth and mycelial abundance. AVRHt1 is inferred to be at the end of scaffold 2 colocalizing with a QTL for radial growth.

TABLE 2. Quantitative trait loci estimated positions on the genetic map, effects, and associated phenotypic variation for multiple traits evaluated on the race $1 \times$ race $23 \mathrm{~N}$ progeny

\begin{tabular}{|c|c|c|c|c|c|c|c|c|}
\hline Trait & Trait expression $^{\mathrm{a}}$ & $\mathrm{LG}^{\mathrm{b}}$ & CI start ${ }^{\mathrm{c}}$ & Peak & $\mathrm{CI}$ end $^{\mathrm{c}}$ & LOD & Effect $^{d}$ & Variation $(\%)$ \\
\hline Compatibility (r/s) & In planta & 4 & 144 & 145 & 146 & 90.1 & 0.9 & 87 \\
\hline Difference on incubation period (dip) & In planta & 4 & 142 & 145 & 150 & 38.8 & 3.0 & 59 \\
\hline Difference on diseased leaf area (ddla) & In planta & 4 & 138 & 145 & 148 & 46.1 & 33.8 & 65 \\
\hline Pigmentation (pgm) & In vitro & 2 & 59 & 64 & 67 & 19.7 & 0.9 & 36 \\
\hline Abundance (abn) & In vitro & 2 & 42 & 69 & 88 & 3.0 & -0.4 & 6 \\
\hline Abundance (abn) & In vitro & 4 & 93 & 138 & 164 & 3.2 & 0.4 & 7 \\
\hline Radial growth (rg) & In vitro & 4 & 117 & 143 & 160 & 3.6 & 5.4 & 8 \\
\hline Pigmentation (pgm) & In vitro & 18 & 13 & 46 & 46 & 4.1 & 0.5 & 9 \\
\hline Radial growth (rg) & In vitro & 18 & 42 & 46 & 46 & 20.7 & 11.8 & 38 \\
\hline
\end{tabular}

a In planta traits were collected on $H t 2$ near isogenic lines in green-house experiments while in vitro traits were collected from LCA petri plates grown in a temperature controlled incubator.

${ }^{\mathrm{b}}$ Linkage group.

c Confidence interval (CI), start and end positions.

${ }^{\mathrm{d}}$ Effect $=$ average phenotype for the race 1 (NY001) allele minus average phenotype for the race $23 \mathrm{~N}(\mathrm{Et} 52 \mathrm{~B})$ allele. 
(Fig. 3). For the QTL on LG 4, strains with the NY001 allele had higher mycelial abundance and larger radial growth than strains with the Et52B allele.

Finally, there was evidence of QTL on LG 18 for sporulation, mycelial abundance, pigmentation, and radial growth. The presence of the race $23 \mathrm{~N}$ ( strain Et52B) genetic regions increased sporulation but reduced mycelial abundance, pigmentation rating, and radial growth (Table 2).

\section{DISCUSSION}

By thoroughly genotyping and phenotyping 13 diverse $S$. turcica field strains and 221 progeny from a cross between race 1 and race $23 \mathrm{~N}$ strains, we identified a robust candidate location for $A V R H t 2$, the known genetic location of AVRHtl (Wu et al. 2013), and the location of nine fitness-related QTL. We first conducted in planta observations on NIL with or without the Ht2 gene with the objective of identifying the location of AVRHt2. The qualitative and quantitative data produced strong evidence for the location of the $A V R H t 2$ locus on LG 4 . The same area of the genome was associated with QTL for two fungal development traits evaluated in vitro: mycelial abundance and radial growth (Fig. 3). The presence of the Et52B (race 23N) chromosomal region allowed the pathogen to overcome the $H t 2$ plant $R$ gene but the colonies produced less abundant mycelium and showed slower radial growth than strains with NY001 (race 1) chromosomal regions.

Although we were unable to confidently place the most significant marker associated with AVRHtl on the genetic map of S. turcica, the physical map suggests that it is likely located toward the end of scaffold 2 in LG 1 . This region also had a QTL for radial growth with a negative effect. These results suggest that radial growth QTL on LG 1 and 4 colocalized with AVRHt1 and AVRHt2, respectively. Although we have not proven that these $A V R$ genes have an effect on fitnessrelated traits (as reflected in radial growth or mycelial abundance), our inability to separate the virulence-related trait from the vegetative growth-related trait by recombination in our progeny strains indicates that the causal genes are either tightly linked or identical. That is, virulence and in vitro developmental traits are controlled by the same chromosomal segment and possibly the same gene or genes. Furthermore, the effect of the radial growth QTL that colocalizes with AVRHt2 (LG 4) and the inferred location of AVRHt1 (LG 1) (Table 2) suggests that strains carrying these genes or alleles might have a competitive advantage for saprophytic growth over strains without the $A V R$ genes or alleles.

In a contrasting case, for the closely related pathogen species Cochliobolus heterostrophus and C. carbonum, the presence of the genes or alleles encoding T-toxin and HC-toxin, respectively, exerted a competitive disadvantage (Klittich and Bronson 1986; Leonard 1977; Welz and Leonard 1993). These two Cochliobolus spp. interact with maize in an inverse gene-for-gene interaction, in which host-specific toxins from the pathogen interact with a susceptibility protein in the host. Conversely, S. turcica interacts with maize in a gene-for-gene interaction in which avirulence factors interact with resistance factors.

Because in vitro fitness may not reflect in planta fitness for this pathogen, we also examined the tradeoffs between virulence on Ht1 and $H t 2$ hosts and aggressiveness on a susceptible host. The presence or absence of the wild-type $A V R H t 1$ or $A V R H t 2$ phenotypes in a given isolate had no significant relationship to that isolate's DLA or IP on the susceptible host. This did not support the hypothesis that the lack of race 1 isolates prior to the widespread deployment of $H t 1$ was due to decreased in planta fitness in lines with mutations in AVRHtl (Ferguson and Carson 2007). When both AVR genes were taken together, however, there was a suggestive relationship between the total number of wild-type $A V R$ genes in a given isolate and its DLA on the susceptible plant $(P=0.056)$ (Fig. 4). This potential relationship certainly merits further study, because many aspects of in planta fitness that could have affected the race distributions of $S$. turcica in the United States could not easily be captured in a greenhouse screen.

Multiple traits were evaluated for the interaction of the progeny with the Ht2 maize gene. All of the significant associations localized AVRHt 2 to LG 4 in overlapping regions spanning 2,8 , or $10 \mathrm{cM}$ (Table 2). LG 4 was composed of scaffolds 8 and 23 of Et28A-v1.0, and the confidence intervals for AVRHt 2 spanned the end of scaffold 8, a predicted gap, and scaffold 23 (Fig. 2). The most significant marker, S8_2099705, was not completely linked to the trait and no obvious candidate gene was present. It is possible that the causative gene for $A V R H t 2$ is not present in the sequenced Et28A strain because it is race $23 \mathrm{~N}$ and expected to be avrHt2, not AVRHt2. The most significant marker successfully predicted the outcome of the interaction with $\mathrm{Ht} 2$ plants for $97 \%$ of the progeny strains (Table 3 ). Strain Et28A, which is known to be race 23N, had the "wrong" SNP marker at S8_2099705, suggesting that the marker is not causal for the interaction. We are working on alternative methods to identify the gene underlying AVRHt2.

AVRHt1 was strongly associated with marker S2_3552054, which aligned to scaffold 2 of Et28A-v1.0. This position on scaffold 2 is in a gene encoding a hybrid PKS-NRPS (JGI protein ID 179218). This was originally identified in a cross-genome comparison of PKS and NRPS proteins in several Cochliobolus strains plus $S$. turcica Et28A (Condon et al. 2013; Ohm et al. 2012). Based on sequence information, we developed an SNP-specific assay for location 3549698 , which had a stop codon in the race 1 strain. This marker perfectly distinguished between strains that caused susceptible or resistant interactions on Pa91Htl maize (Table 3). This assay is now being validated with more $S$. turcica strains. Further molecular characterization of protein JGI 179218 was not the focus of this study and will be published elsewhere.

In vitro developmental traits were evaluated as a proxy for saprophytic growth. Conidiation was the only one of the fungal developmental traits that mapped to a single LG. Multiple QTL were identified for pigmentation, mycelial abundance, and radial growth. The two pigmentation QTL colocalized with mycelial abundance QTL on LG 2 and LG 4, suggesting that pigmentation might be physiologically associated with mycelial abundance. The detection of an additional QTL for mycelial abundance on LG 4, unlinked to pigmentation loci, indicates that there are physiological factors other than pigmentation influencing mycelial abundance. All of the in vitro traits but none of the in planta traits had a QTL in LG 18

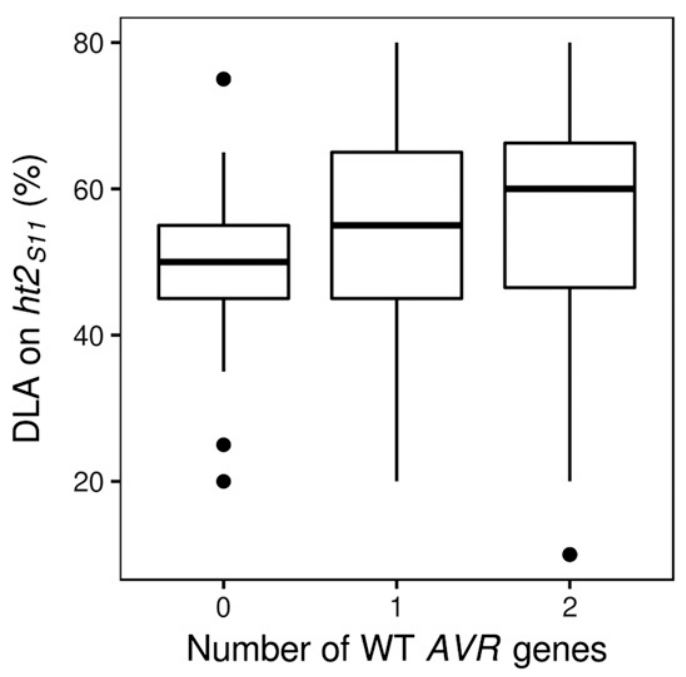

Fig. 4. Boxplot of diseased leaf area (DLA) of NY001 $\times 52 \mathrm{~B}$ isolates on the $h t 2_{S 11}$ near-isogenic line versus the number of (known) wild-type (WT) avirulence $(A V R)$ genes carried by the isolate. Isolates which induced a resistance reaction on both Pa91Htl and $H t 2_{D K 888}$ lines were taken to have two WT $A V R$ genes, those which induced a resistance reaction on only Pa91Htl or $H t 2_{D K 888}$ lines were taken to have one, and lines which did not induce a resistance reaction on either were taken to have zero. 
(Fig. 3). Our results suggest that LG 18 is a major determinant of fungal development that is independent of virulence interactions with $H t 1$ or $H t 2$.

The population set of SNP variants, as well as all of the secondary SNP sets, had only a few thousand markers, despite the 122 million reads used to call the SNP. This was mostly due to the low number of properly segregating polymorphisms. Polymorphisms that segregated 1:1 were present in only approximately $1 \%$ of reads. We tested multiple alternative SNP-calling pipelines but none were able to produce a larger or more marker-dense map than the primary pipeline. The maps produced from haploid SNP calls had most of the same markers as the maps produced from diploid SNP calls and were roughly the same size, suggesting that the presence of heterozygous calls had little overall impact on the final map.

The large number of heterozygous SNP calls was surprising. We believe that these were due to multicopy regions in the $S$. turcica genome. The available evidence related to the heterozygous calls supports a biological explanation and is generally inconsistent with technical or human error (Supplementary File S1). The SNP-specific KASP assay, which was independent of the GBS that initially revealed the heterozygosity, confirmed the heterozygous calls for SNP S2_3549698, and further testing will confirm or reject other calls. Further investigation with independent sequence data sets is needed.

Overall, using a forward genetics approach with thoroughly analyzed and filtered SNP, creating a genetic map, and anchoring it to the physical sequence, we identified regions of the $S$. turcica genome associated with interactions with the maize resistance loci $\mathrm{Htl}$ and Ht2. This study provides molecular markers that identify strains capable of causing disease on plants with the Htl or Ht2 genes. Evidence is presented here that genetic regions that encode virulence to $H t 1$ and $H t 2$ are associated with in vitro developmental traits and possibly with a fitness cost for the pathogen. Finally, evidence is added for the identity of the AVRHtl gene and a genetic location has been identified for the AVRHt2 gene.

\section{ACKNOWLEDGMENTS}

We thank P. Balint-Kurti and G. Bergstrom for providing field strains, T. Jamann for useful review of a draft of this manuscript, and the Joint Genome Institute for supporting $S$. turcica genome sequencing (https://genome.jgi.doe. gov/Settu1/Settu1.home.html). This research was supported by The McKnight Foundation and the U.S. Department of Agriculture under Hatch Project Number 1008676.

\section{LITERATURE CITED}

Akinsanmi, O. A., Chakraborty, S., Backhouse, D., and Simpfendorfer, S. 2007. Passage through alternative hosts changes the fitness of Fusarium graminearum and Fusarium pseudograminearum. Environ. Microbiol. 9: 512-520.

Barnett, D. W., Garrison, E. K., Quinlan, A. R., Stromberg, M. P., and Marth, G. T. 2011. BamTools: A C++ API and toolkit for analyzing and managing BAM files. Bioinformatics 27:1691-1692.

Bates, D., Mächler, M., Bolker, B., and Walker, S. 2015. Fitting linear mixedeffects models using lme4. J. Stat. Softw. 67. doi.org/10.18637/jss.v067.i01

Bradbury, P. J., Zhang, Z., Kroon, D. E., Casstevens, T. M., Ramdoss, Y., and Buckler, E. S. 2007. TASSEL: Software for association mapping of complex traits in diverse samples. Bioinformatics 23:2633-2635.

Broman, K. W., and Sen, S. 2009. A Guide to QTL Mapping with R/qtl. Springer Science+Business Media, New York.

Carson, M. L. 1999. Helminthosporium leaf spots and blights. Pages 15-24 in: Compendium of Corn Diseases, 3rd ed. D. G. White, ed. The American Phytopathological Society, St. Paul, MN.

Catchen, J., Hohenlohe, P. A., Bassham, S., Amores, A., and Cresko, W. A. 2013. Stacks: An analysis tool set for population genomics. Mol. Ecol. 22: 3124-3140.

Chung, C. L., Jamann, T., Longfellow, J., and Nelson, R. 2010. Characterization and fine-mapping of a resistance locus for northern leaf blight in maize bin 8.06. Theor. Appl. Genet. 121:205-227.

Condon, B. J., Leng, Y. Q., Wu, D. L., Bushley, K. E., Ohm, R. A., Otillar, R., Martin, J., Schackwitz, W., Grimwood, J., MohdZainudin, N., Xue, C. S., Wang, R., Manning, V. A., Dhillon, B., Tu, Z. J., Steffenson, B. J., Salamov,
A., Sun, H., Lowry, S., LaButti, K., Han, J., Copeland, A., Lindquist, E., Barry, K., Schmutz, J., Baker, S. E., Ciuffetti, L. M., Grigoriev, I. V., Zhong, S., and Turgeon, B. G. 2013. Comparative genome structure, secondary metabolite, and effector coding capacity across Cochliobolus pathogens. PLoS Genet. 9:e1003233.

DePristo, M. A., Banks, E., Poplin, R., Garimella, K. V., Maguire, J. R., Hartl, C., Philippakis, A. A., del Angel, G., Rivas, M. A., Hanna, M., McKenna, A., Fennell, T. J., Kernytsky, A. M., Sivachenko, A. Y., Cibulskis, K., Gabriel, S. B., Altshuler, D., and Daly, M. J. 2011. A framework for variation discovery and genotyping using next-generation DNA sequencing data. Nat. Genet. 43:491-498.

Elshire, R. J., Glaubitz, J. C., Sun, Q., Poland, J. A., Kawamoto, K., Buckler, E. S., and Mitchell, S. E. 2011. A robust, simple genotyping-by-sequencing (GBS) approach for high diversity species. PLoS One 6:e19379.

Fang, G., Hammar, S., and Grumet, R. 1992. A quick and inexpensive method for removing polysaccharides from plant genomic DNA. Biotechniques 13: 52-56.

Ferguson, L. M., and Carson, M. L. 2007. Temporal variation in Setosphaeria turcica between 1974 and 1994 and origin of races 1, 23, and $23 \mathrm{~N}$ in the United States. Phytopathology 97:1501-1511.

Garrison, E., and Marth, G. 2012. Haplotype-based variant detection from short-read sequencing. Online publication. arXiv:1207.3907v2 [q-bio.GN]

Glaubitz, J. C., Casstevens, T. M., Lu, F., Harriman, J., Elshire, R. J., Sun, Q., and Buckler, E. S. 2014. TASSEL-GBS: A high capacity genotyping by sequencing analysis pipeline. PLoS One 9:e90346.

Huang, Y.-J., Balesdent, M.-H., Li, Z.-Q., Evans, N., Rouxel, T., and Fitt, B. D. L. 2010. Fitness cost of virulence differs between the AvrLml and AvrLm4 loci in Leptosphaeria maculans (Phoma stem canker of oilseed rape). Eur. J. Plant Pathol. 126:279-291.

Klittich, C. J. R., and Bronson, C. R. 1986. Reduced fitness associated with TOX1 of Cochliobolus heterostrophus. Phytopathology 76:1294-1298.

Knox-Davies, P., and Dickson, J. 1960. Cytology of Helminthosporium turcicum and its ascigerous stage, Trichometasphaeria turcica. Am. J. Bot. 47: 328-339.

Langmead, B., and Salzberg, S. L. 2012. Fast gapped-read alignment with Bowtie 2. Nat. Methods 9:357-359.

Leach, J., Lang, B. R., and Yoder, O. C. 1982. Methods for selection of mutants and in vitro culture of Cochliobolus heterostrophus. J. Gen. Microbiol. 128:1719-1729.

Leach, J. E., Cruz, C. M. V., Bai, J. F., and Leung, H. 2001. Pathogen fitness penalty as a predictor of durability of disease resistance genes. Annu. Rev. Phytopathol. 39:187-224.

Lendenmann, M. H., Croll, D., and McDonald, B. A. 2015. QTL mapping of fungicide sensitivity reveals novel genes and pleiotropy with melanization in the pathogen Zymoseptoria tritici. Fungal Genet. Biol. 80:53-67.

Leonard, K. J. 1977. Virulence, temperature optima, and competitive abilities of isolines of races $\mathrm{T}$ and $\mathrm{O}$ of Bipolaris maydis. Phytopathology 67: 1273-1279.

Leonard, K. J., Levy, Y., and Smith, D. R. 1989. Proposed nomenclature for pathogenic races of Exserohilum turcicum on corn. Plant Dis. 73:776-777.

Li, H., and Durbin, R. 2009. Fast and accurate short read alignment with Burrows-Wheeler transform. Bioinformatics 25:1754-1760.

Lim, S. M., Kinsey, J. G., and Hooker, A. L. 1974. Inheritance of virulence in Helminthosporium turcicum to monogenic resistant corn. Phytopathology 64:1150-1151.

Lo Presti, L., Lanver, D., Schweizer, G., Tanaka, S., Liang, L., Tollot, M., Zuccaro, A., Reissmann, S., and Kahmann, R. 2015. Fungal effectors and plant susceptibility. Annu. Rev. Plant Biol. 66:513-545.

Lovell, J. T., Mullen, J. L., Lowry, D. B., Awole, K., Richards, J. H., Sen, S., Verslues, P. E., Juenger, T. E., and McKay, J. K. 2015. Exploiting differential gene expression and epistasis to discover candidate genes for droughtassociated QTLs in Arabidopsis thaliana. Plant Cell 27:969-983.

Luttrell, E. S. 1958. The perfect stage of Helminthosporium turcicum. Phytopathology 48:281-287.

Ma, S. X., Cao, K. K., Liu, N., Meng, C., Cao, Z. Y., Dai, D. Q., Jia, H., Zang, J. P., Li, Z. Y., Hao, Z. M., Gu, S. Q., and Dong, J. G. 2017. The StLAC2 gene is required for cell wall integrity, DHN-melanin synthesis and the pathogenicity of Setosphaeria turcica. Fungal Biol. 121:589-601.

McDonald, B. A. 2015. How can research on pathogen population biology suggest disease management strategies? The example of barley scald (Rhynchosporium commune). Plant Pathol. 64:1005-1013.

McDonald, B. A., and Linde, C. 2002. Pathogen population genetics, evolutionary potential, and durable resistance. Annu. Rev. Phytopathol. 40: 349-379.

Mueller, D., Wise, K., Sisson, A. J., Allen, T. W., Bergstrom, G., Bruce Bosley, D., Bradley, C. A., Broders, K. D., Byamukama, E., Chilvers, M., Collins, A., Faske, T. R., Friskop, A. J., Heiniger, R. W., Hollier, C., Hooker, D. C., Isakeit, T., Jackson-Ziems, T. A., Jardine, D., Kinzer, K., Koenning, S., Malvick, D., McMullen, M., Meyer, R. F., Paul, P., Robertson, A. E., Roth, 
G. W., Smith, D. L., Tande, C. A., Tenuta, A., Vincelli, P., and Warner, F. 2016. Corn yield estimates due to diseases in the United States and Ontario, Canada from 2012 to 2015. Plant Health Prog. 17:211-222.

Ohm, R. A., Feau, N., Henrissat, B., Schoch, C. L., Horwitz, B. A., Barry, K. W., Condon, B. J., Copeland, A. C., Dhillon, B., Glaser, F., Hesse, C. N., Kosti, I., LaButti, K., Lindquist, E. A., Lucas, S., Salamov, A. A., Bradshaw, R. E., Ciuffetti, L., Hamelin, R. C., Kema, G. H. J., Lawrence, C., Scott, J. A., Spatafora, J. W., Turgeon, B. G., de Wit, P. J. G. M., Zhong, S. B., Goodwin, S. B., and Grigoriev, I. V. 2012. Diverse lifestyles and strategies of plant pathogenesis encoded in the genomes of eighteen Dothideomycetes fungi. PLoS Pathog. 8:e1003037.

Perkins, J. M., and Pedersen, W. L. 1987. Disease development and yield losses associated with northern leaf blight on corn. Plant Dis. 71:940-943.

Pietravalle, S., Lemarié, S., and van den Bosch, F. 2006. Durability of resistance and cost of virulence. Eur. J. Plant Pathol. 114:107-116.

R Core Team. 2015. R: A Language and Environment for Statistical Computing. R Foundation for Statistical Computing, Vienna. http://www.r-project.org/

Sen, S., and Churchill, G. A. 2001. A statistical framework for quantitative trait mapping. Genetics 159:371-387.

Ullstrup, A. J., and Miles, S. R. 1957. The effects of some leaf blights of corn on grain yield. Phytopathology 47:331-336.
Untergasser, A., Cutcutache, I., Koressaar, T., Ye, J., Faircloth, B. C., Remm, M., and Rozen, S. G. 2012. Primer3-New capabilities and interfaces. Nucleic Acids Res. 40:e115.

Welz, H. G., and Leonard, K. J. 1993. Phenotypic variation and parasitic fitness of races of Cochliobolus carbonum on corn in North Carolina. Phytopathology 83:593-601.

Wu, D., Mideros, S., Wiesner-Hanks, T., Nelson, R. J., and Turgeon, B. G. 2013. A Setosphaeria turcica secondary metabolite effector prompts a resistance response on $\mathrm{Ht} 1$ maize. In: 27th Fungal Genet. Conf. Asilomar Conference Grounds.

Wu, D., and Turgeon, B. G. 2013. Setosphaeria rostrata: Insights from the sequenced genome of Setosphaeria turcica. Fungal Genet. Biol. 61:158-163.

Xue, C. S., Wu, D. L., Condon, B. J., Bi, Q., Wang, W. W., and Turgeon, B. G. 2013. Efficient gene knockout in the maize pathogen Setosphaeria turcica using Agrobacterium tumefaciens-mediated transformation. Phytopathology 103:641-647.

Zainudin, N. A. I. M., Condon, B., De Bruyne, L., Van Poucke, C., Bi, Q., Li, W., Hofte, M., and Turgeon, B. G. 2015. Virulence, host-selective toxin production, and development of three Cochliobolus phytopathogens lacking the Sfp-type 4'-phosphopantetheinyl transferase Ppt1. Mol. Plant-Microbe Interact. 28:1130-1141. 